\title{
COMMENTARY
}

\section{Anti-platelet drugs, pharmacogenomics and stent thrombosis: What's new?}

\section{A current perspective on the prevention of stent thrombosis}

\section{John Benjamin}

Morningside Medi-Clinic, Sandton, South Africa

Address for correspondence:

PO Box 264

Morningside

Sandton

2057

South Africa

\section{Email:}

practon@icon.co.za

\section{INTRODUCTION}

Stent thrombosis is a catastrophic complication of percutaneous coronary intervention $(\mathrm{PCl})$ and is associated with a mortality of between 25 to $40 \%$. Various trials report the frequency of stent thrombosis as (0.8-2\%). ${ }^{(1,2)}$ Most evaluations however come from registries that are likely to underestimate the real prevalence. ${ }^{(3)}$ The perception that stent thrombosis is very low in all patients has resulted in a lack of adherence to professional guidelines. ${ }^{\left({ }^{4}\right)}$ New data to identify patients at increased risk for stent thrombosis are emerging. The COURAGE and FAME trials should have tempered over-exuberant $\mathrm{PCl}$, and hopefully now the interventionalists will also be proactive in seeking out the patient at higher risk for stent thrombosis. Numerous risk factors for stent thrombosis have been established (Table I). . 5,6$)^{\circ}$

\section{TABLE I: Established risk factors for stent thrombosis}

\begin{tabular}{l|l}
\hline Treatment discontinuation & Stent malpositioning \\
\hline Active smoking & Poor stent apposition \\
\hline Diabetes & Recent MI \\
\hline Bifurcations & Cancer \\
\hline Small arteries & Renal failure \\
\hline Low TIMI flow & Heart failure \\
\hline Increased stent length & Chronic inflammatory diseases \\
\hline Stent type & Polymer type \\
\hline
\end{tabular}

\section{ABSTRACT}

Stent thrombosis is a catastrophic complication of percutaneous coronary intervention $(\mathrm{PCl})$ and is associated with a mortality of 25 to $40 \%$. The perception that stent thrombosis is very low in all patients has resulted in a lack of adherence to professional guidelines. New data to identify patients at increased risk for stent thrombosis are emerging. Clopidogrel is a prodrug and the activation of clopidogrel is dependent on CY2C19. Numerous alleles of CYP2CI9 exist. The allele CYP2CP*2 has been associated with a marked decrease in platelet responsiveness to clopidogrel. Heterozygote carriers of the CYP2C19*2 have a 2.7 fold increased risk and homozygotes a 4.8 fold increased risk of stent thrombosis. Prospective randomised clinical trials will be necessary to determine the efficacy of CYP2C19 genotypedirected therapy in evidence-based clinical decision making. Point-of-care platelet-function tests are becoming available and some centres are now performing such tests on their $\mathrm{PCl}$ patients. The most recent AHA/ACC/SCAI guidelines recommend testing for clopidogrel responsiveness in patients at high risk of sub acute stent thrombosis and recommend increasing the dose of clopidogrel in nonresponders. SAHeart 2010;7:150-153

Treatment discontinuation remains the single most important and potentially preventable risk factor for stent thrombosis. ${ }^{(7)}$

\section{NEW RISK FACTORS FOR STENT THROMBOSIS}

In order to appreciate the effects of variant alleles of the cytochrome P-450 system, the pharmacokinetics and pharmacogenetics of clopidogrel are reviewed.

\section{Clopidogrel pharmacokinetics and the P-450 enzyme system} Most drugs are dependent on the cytochrome P-450 enzymes for their metabolism and elimination and many require bioactivation to form the active compound. Figure I summarises the nomenclature of the cytochrome P-450 system. 


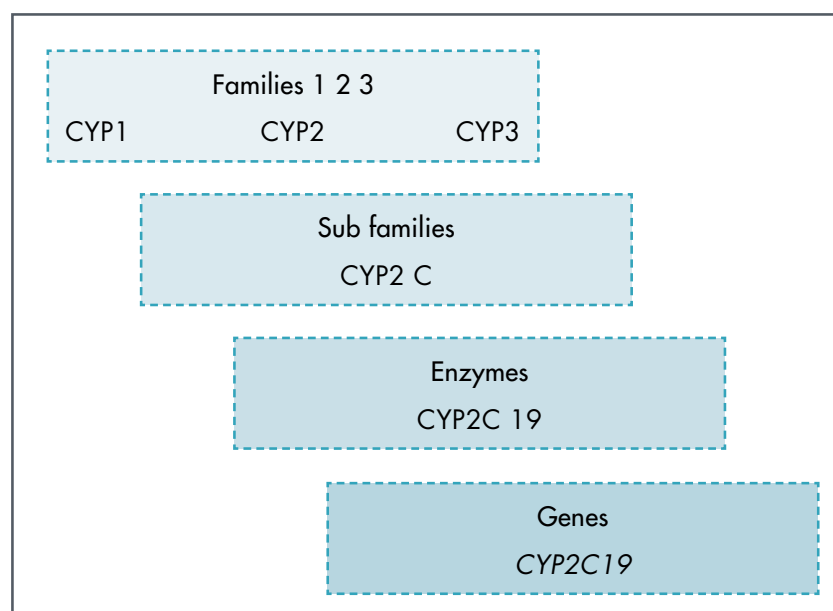

FIGURE I: Nomenclature of the cytochrome P-450 Enzyme System (CYP). CYP-450 enzymes are designated with the abbreviation (CYP), followed by an Arabic numeral indicating the family, a capital letter indicating the subfamily and another numeral for the individual enzyme. The convention is to italicise the name when referring to the gene. For example, CYP2CI9 is the gene that encodes the enzyme CYP2CI9.

A simplified diagram of some CYP-450 enzymes relevant to cardiologists is reviewed in Table 2 .

Three of the many enzymes evolved in drug metabolism are highlighted. CYP2C19 is important in the elimination of proton pump inhibitors (PPIs) and statins but activates the prodrug clopidogrel. CYP3A4/5 metabolises many drugs. CYP3A4/5 is inhibited by grapefruit juice which would result in slower elimination of affected drugs. CYP2C9 is important in warfarin metabolism and elimination.

Clopidogrel is a prodrug. After absorption $85 \%$ is converted into an inactive form by esterases. Only $15 \%$ is then available for conversion to its active metabolite. This conversion process is complex and involves numerous enzymes. CYP2CI9 in particular is required at two separate oxidative steps (Figure 2).

TABLE 2: CYP-450 enzymes relevant to cardiologists

\begin{tabular}{l|l|}
\hline CYP2C19 & Activates: Clopidogrel \\
\hline \multirow{2}{*}{ CYP3A4/5 } & Metabolises: PPIs, statins \\
& Metabolises many drugs: \\
& Lipid soluble statins, calcium channel blockers, \\
\hline CYP2C9 & Amiodarone, PPIs, sildenafil, macrolides \\
\hline
\end{tabular}

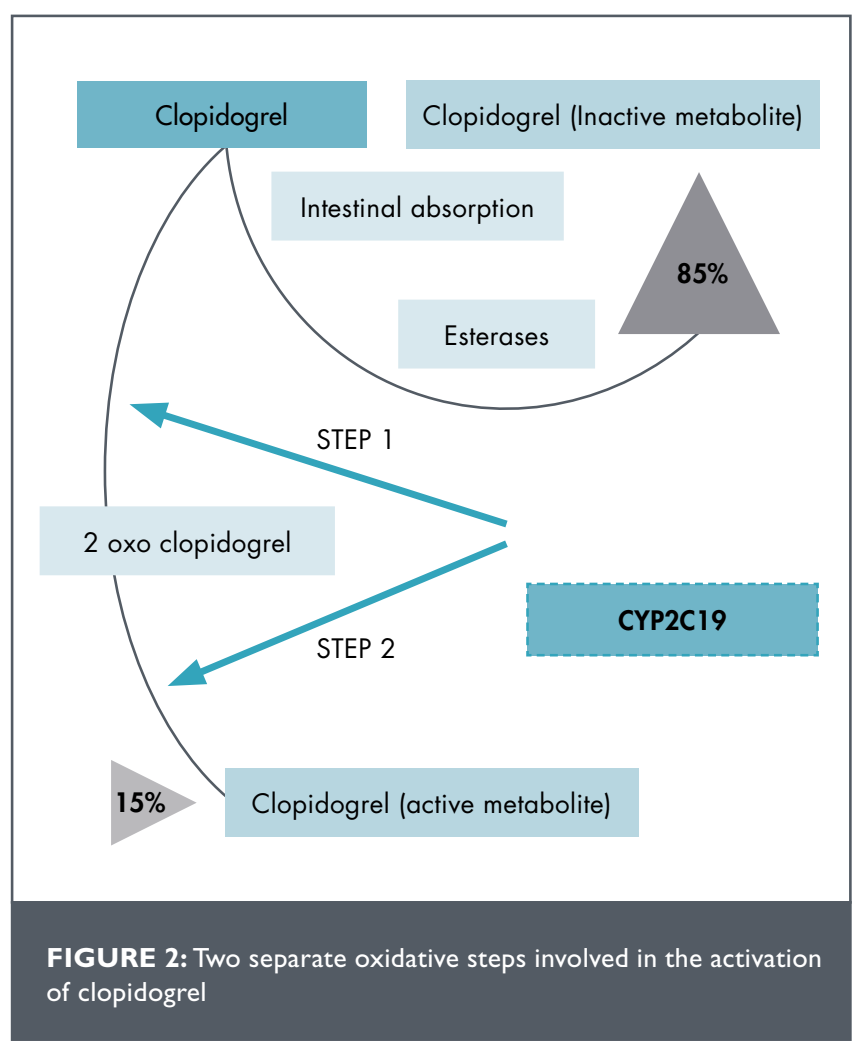

Eighty-five percent of absorbed clopidogrel is converted into an inactive form by esterases. Only I $5 \%$ is then available for conversion to its active metabolite. Clopidogrel activation is dependent on CYP2C19 as it is involved in both of the hepatic oxidative steps.

\section{Basic pharmacogenetics of clopidogrel}

Numerous alleles of CYP2C19 exist. The allele CYP2CP*2 has been associated with a loss of function which results in a marked decrease in platelet responsiveness to clopidogrel. ${ }^{\left({ }^{8}\right)}$ The presence of genetically poorly functioning CYP2C19*2 has been shown to effect clopidogrel activation, especially when it is present in the homozygous state (CYP2C19*2/*2). ${ }^{(9)}$

The FAST-MI study demonstrated an association between stent thrombosis and CYP2C19*/*. The TRITON TIMI 38 study demonstrated an association between stent thrombosis CYP2C19* and CYP2C19*/*.(10,11)

A recent collaborative meta-analysis involving 9 studies presented at the American Heart Association in November 2009 by Mega, revealed that heterozygote carriers of the CYP2C19*2, had a 2.7-fold increased risk and homozygotes a 4.8 -fold increased risk of stent thrombosis. In this meta-analysis, $26 \%$ of the population were heterozygous carriers of the variant allele and $2 \%$ were homozygous carriers. 
Prasugrel (a third generation thienopyridine) does not have the same reliance on CYP2C19 as clopidogrel. In a predefined subgroup analysis of the TRITON-TIMI 38, clinical outcomes of patients on prasugrel were not affected by CYP2C19 polymorphisms. In particular, there was less stent thrombosis. Prasugrel recently received FDA approval based on results of the TRITON-TIMI 38 study. ${ }^{(12)}$ These benefits were, in part, offset by a higher risk of bleeding in prasugrel-treated patients. However an increased bleeding risk did not occur if certain groups were excluded. This subgroup analysis included patients with a history of stroke, age $>75$ years and those with a body weight of less than $60 \mathrm{~kg}$.

Ticagrelor is a reversible oral P2Y 12 receptor antagonist in a new chemical class of anti-platelet agents termed cyclopentyl-triazolopyrimidines that does not require metabolic conversion to an active form. The PLATO trial demonstrated that ticagrelor achieved a statistically significant reduction in the primary efficacy endpoint (cardiovascular death, myocardial infarction or stroke) compared to clopidogrel. ${ }^{(13)}$

Prasugrel and ticagrelor are not yet available in South Africa. This poses the question if we should be testing for CYP2C19* in patients on clopidogrel then. Prospective randomised clinical trials will be necessary to determine the efficacy of CYP2CI 9 genotypedirected therapy in evidence-based clinical decision-making. Rapid genetic testing for the CYP2C19*2 variant is not available but is being developed.

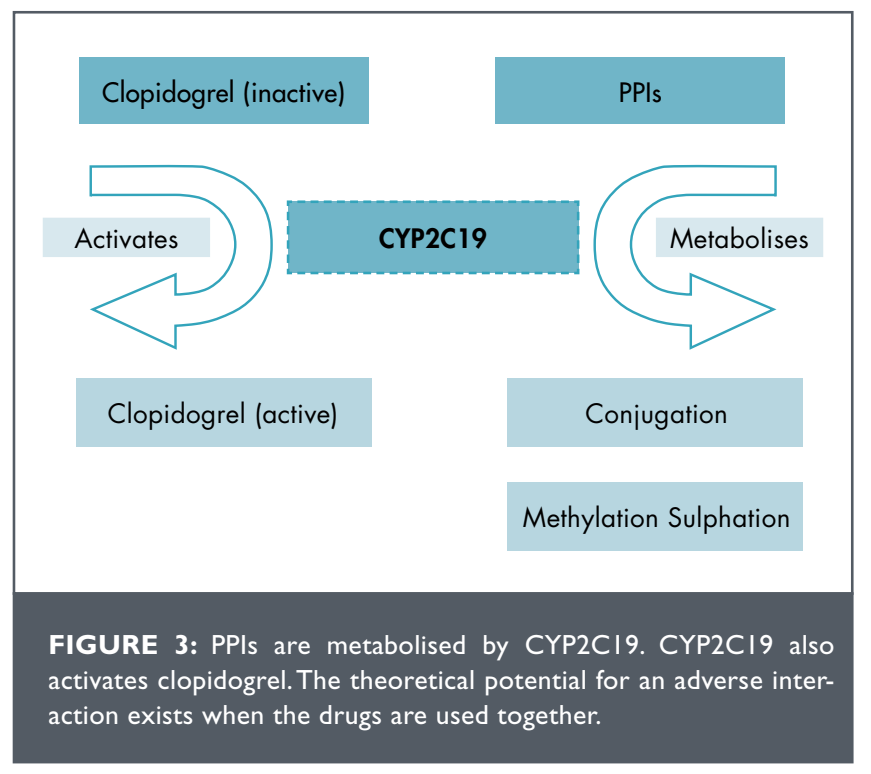

\section{Drug interactions}

PPIs are metabolised by CYP2C19 and may therefore compete for CYP2C19 when used in conjunction with clopidogrel.

There have been numerous observational studies which suggest that the clopidogrel and PPI interaction can lead to adverse cardiovascular outcomes ${ }^{(14)}$ (Figure 3). Omeprazole, in particular, has been incriminated while pantoprazole has reputedly less interaction with PPIs. ${ }^{(15)}$ In the subgroup analyses of the CREDO andTRITON trials $^{(16,17)}$ no interaction was found. Preliminary analysis of data from the COGENT trial, a trial dedicated to specifically examining clopidogrel with a PPI vs placebo, failed to suggest a negative interaction. There is therefore conflicting evidence, but as some concern still exists prescribers should avoid the use of PPIs if possible. On November 17, 2009, the FDA updated the clopidogrel label with new warnings on omeprazole and other drugs that inhibit the CYP2C19 enzyme that could interact with clopidogrel.

High on-treatment platelet reactivity

There are consistent data that patients who have persistently increased platelet reactivity despite clopidogrel therapy have an increased risk of ischaemic events. Recent findings have shown that a considerable number of patients who are resistant to clopidogrel may also be resistant to aspirin, which further increase their risk of stent thrombosis. ${ }^{(18)}$

Should we be measuring platelet function in patients who are taking clopidogrel after stent implantation? Should the dose of clopidogrel be increased in patients resistant to standard doses? It is interesting that the recently presented CURRENT OASIS-7 study (presented at the European Society of Cardiology 2009) revealed that a loading dose of $600 \mathrm{mg}$ together with a higher maintenance dose of clopidogrel (I50mg for one week after PCl) was associated with less stent thrombosis. There was no increased risk of bleeding when the TIMI major bleeding criteria were used. There was however a small increased risk of red cell transfusion.

Point-of-care platelet-function tests are becoming available and some centres are now performing such tests on their $\mathrm{PCl}$ patients. Unfortunately, there is no evidence that modifying the treatment of a hypo-responsive patient improves outcomes. Numerous trials are being conducted in this regard:

- GRAVITAS (Gauging Responsiveness with a Verify Now AssayImpact on Thrombosis and Safety) trial, randomising patients deemed non-responsive to clopidogrel on point-of-care testing to receive higher dosing of clopidogrel. ${ }^{(19)}$ 
- TRIGGER PCl is assessing the efficacy of prasugrel versus clopidogrel for the reduction of adverse cardiovascular outcomes in drug-eluting stent (DES) patients with high platelet reactivity on clopidogrel, using the Verify Now test.

ARCTIC double randomisation of a monitoring adjusted antiplatelet treatment versus a common anti-platelet treatment for DES-implantation and interruption versus continuation of double anti-platelet therapy.

\section{CONCLUSION}

The most recent AHA/ACC/SCAI guidelines recommend testing for clopidogrel responsiveness in patients at high risk of sub-acute stent thrombosis and recommend increasing the dose of clopidogrel in non-responders. The United Kingdom's National Institute for Health and Clinical Excellence (NICE) has issued its provisional guidance on the use of prasugrel. The guidelines include patients undergoing primary $\mathrm{PCl}$ for ST-segment elevation MI, subsequent to stent thrombosis having occurred on clopidogrel treatment and in patients with diabetes mellitus. ${ }^{(20)}$

In France prasugrel is not available but compassionate usage has been increasing (Montalescot - personal communication).

Prasugrel and ticagrelor are not yet available in South Africa, so what should we do?

- A conservative approach would be to initially accept the results of the CURRENT OASIS-7 study and double the loading dose of clopidogrel to $600 \mathrm{mg}$ and increase the maintenance dose of clopidogrel to $150 \mathrm{mg}$ for one week, while waiting for the results of the randomised trials.

- A more proactive approach would be to measure platelet function and increase the dose of clopidogrel in non-responders and if there is no improvement in response, consider obtaining compassionate use of prasugrel.

It should be emphasised however, that when stent thrombosis has occurred on clopidogrel treatment, serious consideration should be given to changing treatment to prasugrel.

\section{REFERENCES}

I. Tsuchida K, Morger C, Boersma E, et al. Incidence and correlates of drugeluting stent thrombosis in routine clinical practice. 4-year results from a large 2-institutional cohort study. J Am Coll Cardiol 2008;52: I 134-I I 40.

2. Camenzind E, Steg PG, Wijns W. Stent thrombosis late after implantation of first generation drug-eluting stents: A cause for concern. Circulation 2007; I I5: |440-1 455.

3. Montalescot G, Hulot J-S, Collet, J-P. Stent thrombosis: Who's guilty? Eur Heart J 2009:30:2685-2688.

4. Hannan EL, Racz MJ, Gold J, et al. Adherence of catheterisation laboratory cardiologists to American College of Cardiology/American Heart Association guidelines for percutaneous coronary interventions and coronary artery bypass graft surgery: What happens in actual practice? Circulation 2009;121:267-275.

5. Airoldi F, Colombo A, Morici N, et al. Incidence and predictors of drug-eluting stent thrombosis during and after discontinuation of thienopyridine treatment. Circulation 2007; 1 16:745-754.

6. Van Werkum JW, Heestermans AA, et al. Predictors of coronary stent thrombosis: The Dutch Stent Thrombosis Registry. J Am Coll Cardiol 2009;53:1399-1409.

7. Schulz S, Schuster T, Mehilli J, et al. Stent thrombosis after drug-eluting stent implantation: Incidence, timing, and relation to discontinuation of Clopidogrel therapy over a 4-year period. Eur Heart J 2009;30:27|4-2721.

8. Angiolillo DJ, Fernandez Ortiz A, Bernardo E, et al. Variability in individual responsiveness to Clopidogrel. Clinical implications, management, and future perspectives. J Am Coll Cardiol 2007;49:1505- 16.

9. Fontana P, Hulot JS, De Moerloose P, et al. Influence of CYP2CI9 and CYP3A4 gene polymorphisms on Clopidogrel responsiveness in healthy subjects. J Thromb Haemost 2007;5:2153-5.

10. Mega JL, Close SL, Wiviott SD, et al. Cytochrome P450 polymorphisms and response to Clopidogrel. N Engl J Med 2009;360:354-62.

1।. Montalescot G, Wiviott SD, Braunwald E, et al. Prasugrel compared with Clopidogrel in patients undergoing percutaneous coronary intervention for ST-segment elevation myocardial infarction (TRITON-TIMI 38): double-blind, randomised controlled trial. Lancet 2009; 373:723-31.

12. Wiviott SD, Braunwald E, McCabe CH, et al. Prasugrel versus Clopidogrel in patients with acute coronary syndromes. N Engl J Med, 2007; 357, 200 I-20 I 5.

13. Wallentin L, Becker RC, Budaj A, et al. Ticagrelor versus Clopidogrel in patients with acute coronary syndromes. N Engl J Med 2009;36 I: 1045- 1057.

14. Aubert RE, Epstein RS, et al. Proton Pump Inhibitors Effect on Clopidogrel Effectiveness: The Clopidogrel Medco Outcomes Study. Circulation. 2008; 118:S_815.

15. Juurlink DN, Gomes T, Ko DT, et al. A population-based study of the drug interaction between proton pump inhibitors and Clopidogrel. CMAJ 2009;180:713-8.

16. Dunn SP, Macaulay TE, et al. Baseline proton pump inhibitor use is associated with increased cardiovascular events with and without the use of Clopidogrel in the CREDO Trial Circulation 2008;1 18:S_81.

17. O'Donague M, Braunwald E, et al. Pharmacodynamic effect and clinical efficacy of Clopidogrel and Prasugrel with or without a proton-pump inhibitor: An analysis of two randomised trials. Lancet:2009;374:989-997.

18. Gori AM, Marcucci R, Migliorini A, et al. Incidence and clinical impact of dual non-responsiveness to aspirin and Clopidogrel in patients with drug-eluting stents. J Am Coll Cardiol 2008;52:734.

19. Price MJ, Berger PB, Angiolillo DJ, et al. Evaluation of individualised Clopidogrel therapy after drug-eluting stent implantation in patients with high residual platelet reactivity: Design and rationale of the GRAVITAS trial. Am Heart J. 2009 May; |57(5):818-24, 824.el.

20. Wiviott SD, Braunwald E, Angiolillo DJ, et al. Greater clinical benefit of more intensive oral anti-platelet therapy with Prasugrel in patients with diabetes mellitus in the trial to assess improvement in therapeutic outcomes by optimising platelet inhibition with Prasugrel: Thrombolysis in myocardial infarction 38. Circulation 2008; I18:1626-1636. 